\title{
Evaluation of Endometrial Changes with Transvaginal Power Doppler Ultrasound and Comparing Them with Endometrial Sampling in Breast Cancer Patients Using Tamoxifen
}

\author{
Önder Sakin 1, Bülent Kars ${ }^{1}$, () Orhan Ünal1 , Engin Ersin Şimşek², Halim Ömer Kaşıkçı²,

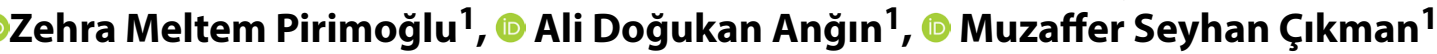 \\ ${ }^{1}$ Dr Lutfi Kirdar Kartal Training and Research Hospital Obstetrics and Gynecology Clinic, Istanbul, Turkey \\ ${ }^{2}$ Dr Lutfi Kirdar Kartal Training and Research Hospital Family Medicine Clinic, Istanbul, Turkey
}

\begin{abstract}
Introduction: The most unwanted complication of tamoxifen is the development of endometrial carcinoma. In this study, we used transvaginal power Doppler ultrasound to determine endometrial vascular changes and compared them with the results of endometrial sampling. The diagnostic value of transvaginal power Doppler ultrasound was examined.

Methods: One hundred eleven breast cancer patients were included in the study. Endometrial, uterine, adnexal, and vascular evaluations were performed. Endometrial sampling was recommended to patients whose endometrial thickness was over $5 \mathrm{~mm}$ and was done in 73 patients.

Results: Group 1 ( $n=62$ ) consisted of patients receiving tamoxifen and group 2 ( $n=49)$ consisted of patients who were not using tamoxifen. There were four endometrial polyps (5.5\%) in the sample results in Group 2. Twenty patients (14 tamoxifen using and 6 non-tamoxifen using) had abnormal vascular changes on power Doppler examination but none of them had any malignant changes. There were abnormal vascular patterns on power Doppler examination in all of the patients detected with endometrial polyps. Three of them had benign measurable Doppler flow characteristics while one of them had non-measureable flows. All of the patients detected to have an endometrial polyp were pre-menopausal and did not use tamoxifen.

Discussion and Conclusion: Transvaginal power Doppler ultrasound is an effective method for determining vascular changes in the endometrium. However, it is not enough for defining the lesion accurately in breast cancer patients. Moreover, it is not useful in determining which patients require invasive intervention.

Keywords: Breast cancer; endometrial cancer; tamoxifen; ultrasonography Doppler.
\end{abstract}

B reast cancer is the most common malignancy in women and constitutes one third of the malignancies in women. Endometrial cancer is the most common type of female genital malignancies and constitutes nearly half of all genital malignancies ${ }^{[1-3]}$.

Tamoxifen is the most commonly used hormonal agent in breast cancer treatment. It is very important to follow up patients receiving tamoxifen for its possible effects on long term usage ${ }^{[4]}$.

The most unwanted side effect of tamoxifen is the development of endometrial cancer ${ }^{[5]}$.

Additionally, uterine sarcoma, malignant mixed Mullerian tumor, and uterine metastasis were reported in patients using tamoxifen. Furthermore, premalignant and benign

Correspondence (iletişim): Önder Sakin, M.D. Dr Lutfi Kirdar Kartal Training and Research Hospital Obstetrics and Gynecology Clinic, Istanbul, Turkey

Phone (Telefon): +90 5326715666 E-mail (E-posta): sakin-onder@hotmail.com

Submitted Date (Başvuru Tarihi): 17.03.2018 Accepted Date (Kabul Tarihi): 19.06.2018

Copyright 2018 Haydarpaşa Numune Medical Journal

This is an open access article under the CC BY-NC license (http://creativecommons.org/licenses/by-nc/4.0/). 
changes such as, endometrial hyperplasia, endometrial thickening, ovarian cysts, endometrial polyps, increase in the size of uterine fibroids, flare up or regression in endometriotic foci, and cervical and vaginal changes may occur ${ }^{[4-6]}$.

It is imperative to detect these premalignant and malignant changes early using minimally invasive techniques in order to avoid unnecessary invasive techniques and procedures.

Power Doppler ultrasound assessment gives us useful information about these patients and secondary information about vascular changes in the tumoral structures that are detected ${ }^{[7]}$.

Our aim was to investigate uterine vascular and endometrial structural and vascular changes with power Doppler ultrasound in patients with breast cancer and to evaluate the success of detecting benign or malignant lesions in tamoxifen users and non-users.

\section{Materials and Methods}

Among patients who were treated for breast cancer between 1993 and 2010 at our hospital, 111 patients agreed to participate in the study. We included all patients who had been diagnosed with breast cancer and who had not undergone a hysterectomy operation. We excluded women with severe systemic medical conditions (heart failure, uncontrolled severe hypertension, etc.), cervical stenosis, acute cervicitis / vaginitis, or lidocaine allergy.

This prospective case control study was performed with the permission of the local ethics control committee and informed consent was obtained from all patients. Transvaginal ultrasound, color Doppler, and power Doppler evaluations were done and findings were noted. Uterine volume, adnexal and endometrial features (thickness, homogeneity, fluid collection, cystic changes, polyps view), abnormal vascular forms (single vessel pattern, scattered vascular pattern, multiple vascular pattern), uterine artery Doppler pulsatility index (PI), resistance index (RI), and systole/diastole ratio $(S / D)$ measurements were done routinely to all patients.

Endometrial biopsies were performed under local anesthesia in patients who agreed to undergo the procedure and in patients with an endometrial thickness above 5 millimeters. Patients were taken to the gynecological examination table in the dorsal lithotomy position. All women underwent bimanual pelvic examination to determine the size and position of uterus. Cervix was exposed with a bivalve speculum and washed with povidine-iodine solution. $2 \mathrm{ml} \mathrm{2 \%} \mathrm{lidocaine} \mathrm{(lekaine} \mathrm{Ampoule,} \mathrm{IE} \mathrm{Ulagay,} \mathrm{Istanbul,}$ Turkey) was injected into the cervix at the 4 and 8 o'clock positions and at the depth of $2-3 \mathrm{~cm}$ after confirming the tip of needle was not inside a vessel lumen. Five minutes were allowed to pass to ensure that the anesthetic effect of lidocaine had started. The biopsies were taken with a Pipelle cannula or uterine curettes after dilatation of the cervix. Specimens were pathologically delivered in $10 \%$ buffered formaldehyde and paraffin blocks were formed afterwards. Selected paraffin sections of $4 \mu \mathrm{m}$ thicknesses were evaluated by using an Olympus microscope (Olympus Life Science Solutions, Florida, America), at x10-x400 power magnifications. The pathological results of endometrial biopsies were recorded.

Patient age, marital status, socioeconomic status, educational status, working status, body mass index, habits, personal history, family history, pregnancy history, age of menarche, duration of menopause, existence of postmenopausal bleeding, tamoxifen usage, history of chemotherapy or radiotherapy were all recorded from the patients' files.

Transvaginal ultrasound, color Doppler, and power Doppler evaluations were done with a $7 \mathrm{MHz}$ (bandwidth 4.3-8.6 $\mathrm{MHz}$, viewing angle width $121^{\circ}$ ) 70 ECLA vaginal probe in an Siemens Accuson Antares ultrasound machine (Siemens Healthcare Diagnostics, Tarrytown, America). Sagittal sections of the uterus were evaluated by placing the vaginal probe to the posterior fornix. Morphologies of transverse, longitudinal, and antero-posterior planes were evaluated. Longitudinal length of the uterus was measured as the distance between the apex of uterus and the end of the cervix by imaging the fundus and the cervix in the same plane; and the length was recorded in millimeters $(\mathrm{mm})$. Fundal and cervical antero-posterior diameters were measured. The uterus was imagined as an ellipsoid shape and the volume was calculated with the D1xD2xD3x0.52 formula. Presence of myomas and adenomyosis were recorded. Pattern of endometrial echo with continuity of subendometrial halo, density, homogeneity, endometrial contour, presence of cystic appearances, liquid accumulation, and presence of polyp images were evaluated. In order to investigate whether tamoxifen caused any changes in the uterine blood flow, $\mathrm{PI}, \mathrm{RI}, \mathrm{S} / \mathrm{D}$ values from uterine arteries was detected with color Doppler and vascular changes were evaluated with power Doppler. Lowest PI and RI values in the vessels were recorded using at least three consecutive waveforms. All evaluations were made by the same physician.

SPSS for Windows v. 15.0 program was used for statistical analysis. Dependent variables in the two groups were evaluated with the student's $t$ test; quantitative data were evaluated with chi-square test. Statistical significance was set at $p<0.05$. 


\section{Results}

$84(75.7 \%)$ of the 111 patients were menopausal and the mean menopause duration was $4.2 \pm 7.42$ years. During the course of the study, one of the patients had postmenopausal bleeding and biopsy revealed a benign diagnosis.

The mean duration after diagnosis of breast cancer was $30.3 \pm 23.7$ months (minimum 1 month and maximum 104 months). 62 (55.9\%) patients were on tamoxifen and the mean tamoxifen usage duration was $21.6 \pm 1.7$ months (minimum 1 month and maximum 60 months).

There were no significant differences in uterine artery Doppler flow examinations, i.e., $\mathrm{PI}, \mathrm{RI}, \mathrm{S} / \mathrm{D}$ changes between tamoxifen users and non-users (left uterine PI mean: $2.57 \pm 0.87$ and $2.33 \pm 0.70$, RI mean: $0.86 \pm 0.06$ and $0.84 \pm 0.79$, S/D mean: $8.80 \pm 3.53$ and $8.16 \pm 3.32$; right uterine PI mean: $2.58 \pm 0.87$ and $2.41 \pm 0.78$, RI mean: $0.86 \pm 0.07$ and $0.84 \pm 0.07$, S/D mean: $9.05 \pm 3.55$ and $8.01 \pm 3.29$, respectively). Twenty patients (18.01\%) had meaningful vascular changes with abnormal vascular pattern. Vessel changes were measurable with flow Doppler examinations in 16 (14.41\%) patients and their PI, Rl, and S/D measurements were not statistically different (Table 1 ).

Tamoxifen had significant effects on the uterus, such as development of cystic changes and variation of the uterine homogeneity. However, it was determined that tamoxifen does not cause significant changes in endometrial fluid collection and endometrial polyp appearance (Table 2).

We determined that there were no significant changes in uterus volume and endometrial thickness in premenopausal tamoxifen users, whereas there were significant increases in the uterus volume and endometrial thickness in postmenopausal tamoxifen users. The uterine volume was found to be $67.460 \pm 39.091 \mathrm{~mm}^{3}$ in the tamoxifen group and $40.240 \pm 25.909 \mathrm{~mm}^{3}$ in the non-tamoxifen group ( $p=0.001)$. The endometrial thickness was found to be $8.69 \pm 5.22 \mathrm{~mm}$ in the tamoxifen group and $5.32 \pm 3.14$ $\mathrm{mm}$ in the non-tamoxifen group $(\mathrm{p}=0.001)$.
Table 1. The results of the patients using tamoxifen or not using tamoxifen

Tamoxifen usage Patient number Mean

Age (years)

Gravida

Vascular $\mathrm{PI}^{*}$

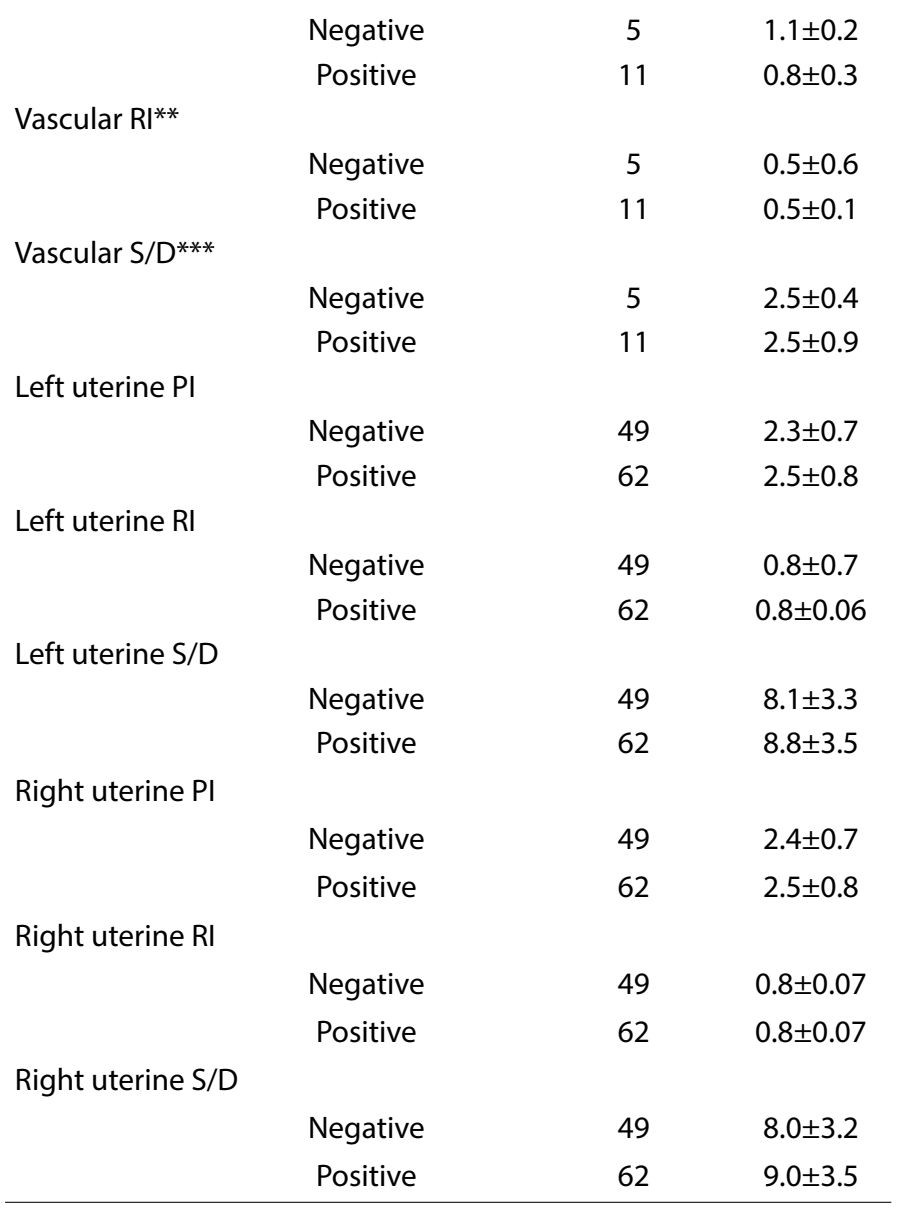

*PI: pulsatility index; ${ }^{* *} \mathrm{RI}$ : resistance index; ${ }^{* *}$ S/D: systole/diastole ratio.

Table 2. Endometrial changes in ultrasound after using tamoxifen

\begin{tabular}{lccccc}
\hline & \multicolumn{2}{c}{ Using tamoxifen $(\mathbf{n}=\mathbf{6 2})$} & \multicolumn{1}{c}{ Not using tamoxifen $(\mathbf{n}=\mathbf{4 9})$} & $\mathbf{p}$ \\
\hline Endometrial homogeneity & Regular & Irregular & Regular & Irregular & \\
& 39 & 22 & 45 & 4 & $\mathrm{p}=0.001$ \\
Endometrial cystic changes & Positive & Negative & Positive & Negative & \\
& 24 & 38 & 0 & 49 & $\mathrm{p}=0.001$ \\
Endometrial fluid collection & Positive & Negative & Positive & Negative & \\
Endometrial polyp view & 3 & 59 & 2 & 47 & $\mathrm{p}=0.1$ \\
& Positive & Negative & Positive & Negative & $\mathrm{p}=0.1$ \\
\hline
\end{tabular}


Seventy-three (65.76\%) biopsies were performed in 111 patients. Thirty-eight (34.23\%) patients did not go through the biopsy and 29 (26.12\%) of them had an endometrial thickness less than $5.5 \mathrm{~mm}$. The remaining ( 9 patients) preferred follow up although they had an endometrial thickness of $6 \mathrm{~mm}$ or more.

The mean endometrial thickness in tamoxifen users was $8.46 \mathrm{~mm}$ in premenopausal and $8.69 \mathrm{~mm}$ in the postmenopausal group.

Transvaginal power Doppler ultrasound examinations were performed in all patients. Twenty patients had meaningful vascular changes with abnormal vascular pattern. Vessel changes were measurable with flow Doppler examination in 16 patients and 4 of these were identified as pathological endometrial polyps. Vascular changes were observed more among the tamoxifen users. Two of these vascular changes were 'single vessel pattern' and the other two were 'scattered vessel pattern.' All endometrial sampling results were benign (Table 3 ).

There were no significant differences in color Doppler flow examinations between the patients. Only four of them had significant changes in power Doppler ultrasound findings. Two endometrial polyps were detected in single vessel pattern. Scattered vessel pattern did not appear consistent with any endometrial pathology such as endometrial hyperplasia. All the patients with endometrial polyps $(n=4)$ were in non-tamoxifen group, in premenopausal state, aged under 43 years, with BMls less than 28 , and with endometrial thicknesses above $10 \mathrm{~mm}$. Abnormal vessel changes were observed in four patients and these changes were measurable in three of them and vessel flow patterns revealed benign characteristics.

\section{Discussion}

Our study revealed that gray-scale transvaginal ultrasound evaluation and power Doppler vascular evaluations have statistically significant changes in tamoxifen users such as, uterine volume, endometrial thickness, irregular endometrial homogeneity, endometrial cystic changes, abnormal vascularity of endometrium $(p<0.001)$.
The endometrial changes caused by tamoxifen have long been acknowledged. Achiron et al. ${ }^{[8-9]}$ evaluated endometrial changes with transvaginal Doppler ultrasound in 45 postmenopausal patients using tamoxifen and they found that tamoxifen causes abnormal thickening, non-homogeneous endometrial pattern, and multiple cystic formations. They stated that these changes mimic neoplasia. In our study, we detected the same cystic formations $(p<0.001)$ and irregular endometrial homogeneity $(p<0.001)$ significantly more in the tamoxifen using group of which all were asymptomatic except one, but all were evaluated as pathologically benign.

Again, in a study by Alcazar et al., ${ }^{[10]}$ successful results were reported. Power Doppler flow is described as 3 types; single-vessel pattern, scattered wave, and multiple wave patterns. Of the 91 cases in their study, endometrial cancer was detected in 33 (36\%), endometrial polyp in 37 (41\%), endometrial hyperplasia in $14(15 \%)$, and cystic atrophy in $7(8 \%)$ patients. $97.1 \%$ of the polyps showed single-vessel pattern, $72.7 \%$ of hyperplasia cases showed scattered pattern and $81.3 \%$ of carcinomas had multiple wave pattern. There were no false positive cases in multiple wave patterns, seven false positive cases in single wave pattern, and nine false positive cases in scattered wave pattern. The transvaginal power Doppler ultrasound study of blood flow was found to be useful in differential diagnosis. In our study 20 of the patients had irregular vascular patterns as described in the study, but 16 of them had measureable vascular structures. Eleven tamoxifen using patients had abnormal vascular formations with mean RI value of 0.55 and no malignancy was detected. Five non-tamoxifen using patients with abnormal vascular forms had a mean RI value 0.59 and no malignancy was detected. Four of them had significant changes as described. Two endometrial polyps were detected in single vessel pattern. Scattered vessel pattern did not appear consistent with any endometrial pathology such as endometrial hyperplasia.

In some previous studies, it is stated that endometrial vascular structures in ultrasound scans can help diagnosis with high accuracy rates ${ }^{[11,12]}$. Successful algorithms for identifying endometrium cancer are also described ${ }^{[13]}$.

Table 3. Endometrial sampling results

\begin{tabular}{lccccc}
\hline & \multicolumn{2}{c}{ Endometrial sampling } & & \multicolumn{2}{c}{ Histopathology result } \\
\cline { 2 - 3 } & Positive & Negative & & Benign & Malign \\
\hline Using tamoxifen $(n=62)$ & 48 & 14 & 48 & 0 & $p=0.02$ \\
Not using tamoxifen $(n=49)$ & 25 & 24 & 25 & 0 & 0 \\
\hline
\end{tabular}


But the same success has not been supported in all studies. They thought that vascular findings depend on tumor characteristics and the sample size of the studies ${ }^{[14,15]}$. And some studies suggest that power Doppler examinations do not improve the diagnostic efficiency of malignancies ${ }^{[16]}$. Dueholm et al. ${ }^{[17]}$ examined the efficiency of power Doppler sonography and stated that it is still uncertain if it is best to correlate different vascular patterns with malignancy.

We do not know exactly whether it shows changes according to tumor characteristics or according to different group of patients. We wanted to examine whether this is meaningful in assessing vascular changes in breast cancer patients. There are no studies that have previously examined uterine and endometrial changes with power Doppler ultrasonography in patients with breast cancer.

Atypical vascularization was observed more frequently in the tamoxifen user group in our study. The $\mathrm{PI}, \mathrm{RI}, \mathrm{S} / \mathrm{D}$ values in these vessels was unaffected and the pathology results did not differ and no malignancy was found.

It is clear that tamoxifen causes significant changes in the uterus and endometrium. But it is not yet possible to pinpoint and foresee precisely which of these changes are associated with malignancy.

\section{Conclusion}

Transvaginal power Doppler ultrasound is an effective method to determine vascular changes in the endometrium. However, it is not suitable for defining the lesion correctly in breast cancer patients. It is also not useful in determining patients in whom we should act invasively.

Peer-review: Externally peer-reviewed.

Authorship Contributions: Concept: O.S., B.K.; Design: O.S., B.K., O.U., Z.M.P.; Data Collection or Processing: O.S., B.K., O.U., Z.M.P.; Analysis or Interpretation: E.E.S., H.O.K.; Literature Search: O.S., A.D.A., M.S.Ç.; Writing: O.S., A.D.A., M.S.Ç.

Conflict of Interest: None declared.

Financial Disclosure: The authors declared that this study received no financial support.

\section{References}

1. Siegel RL, Miller KD, Jemal A. Cancer Statistics, 2017. CA Cancer J Clin 2017;67:7-30. [CrossRef]

2. Torre LA, Bray F, Siegel RL, Ferlay J, Lortet-Tieulent J, Jemal A. Global cancer statistics, 2012. CA Cancer J Clin 2015;65:87-108.

3. Torre LA, Siegel RL, Ward EM, Jemal A. Global cancer incidence and mortality rates and trends-an update. Cancer Epidemiol Biomarkers Prev 2016;25:16-27. [CrossRef]
4. Crew KD, Albain KS, Hershman DL, Unger JM, Lo SS. How do we increase uptake of tamoxifen and other anti-estrogens for breast cancer prevention? NPJ Breast Cancer 2017;3:20.

5. Rosell J, Nordenskjöld B, Bengtsson NO, Fornander T, Hatschek $\mathrm{T}$, Lindman $\mathrm{H}$, et al. Long-term effects on the incidence of second primary cancers in a randomized trial of two and five years of adjuvant tamoxifen. Acta Oncol 2017;56:614-7.

6. Assikis VJ, Neven P, Jordan VC, Vergote I. A realistic clinical perspective of tamoxifen and endometrial carcinogenesis. Eur J Cancer 1996;32A:1464-76. [CrossRef]

7. Kabil Kucur S, Temizkan O, Atis A, Gozukara I, Uludag EU, Agar $S$, et al. Role of endometrial power Doppler ultrasound using the international endometrial tumor analysis group classification in predicting intrauterine pathology. Arch Gynecol Obstet 2013;288:649-54. [CrossRef]

8. Cohen I, Beyth Y, Azaria R, Flex D, Figer A, Tepper R. Ultrasonographic measurement of endometrial changes following discontinuation of tamoxifen treatment in postmenopausal breast cancer patients. BJOG 2000;107:1083-7. [CrossRef]

9. Achiron R, Grisaru D, Golan-Porat N, Lipitz S. Tamoxifen and the uterus: an old drug tested by new modalities. Ultrasound Obstet Gynecol 1996;7:374-8. [CrossRef]

10. Alcazar JL, Galvan R. Three-dimensional power Doppler ultrasound scanning for the prediction of endometrial cancer in women with postmenopausal bleeding and thickened endometrium. Am J Obstet Gynecol 2009;200:44.e1-6. [CrossRef]

11. Opolskiene G, Sladkevicius $P$, Valentin L. Prediction of endometrial malignancy in women with postmenopausal bleeding and sonographic endometrial thickness $\geq 4.5 \mathrm{~mm}$. Ultrasound Obstet Gynecol 2011;37:232-40. [CrossRef]

12. de Kroon CD, Hiemstra E, Trimbos JB, Jansen FW. Power Doppler area in the diagnosis of endometrial cancer. Int J Gynecol Cancer 2010;20:1160-5. [CrossRef]

13. Dueholm M, Møller C, Rydbjerg S, Hansen ES, Ørtoft G. An ultrasound algorithm for identification of endometrial cancer. Ultrasound Obstet Gynecol 2014;43:557-68. [CrossRef]

14. Epstein E, Van Holsbeke C, Mascilini F, Måsbäck A, Kannisto P, Ameye $L$, et al. Gray-scale and color Doppler ultrasound characteristics of endometrial cancer in relation to stage, grade and tumor size. Ultrasound Obstet Gynecol 2011;38:586-93.

15. Saarelainen SK, Vuento MH, Kirkinen P, Mäenpää JU. Preoperative assessment of endometrial carcinoma by three-dimensional power Doppler angiography. Ultrasound Obstet Gynecol 2012;39:466-72. [CrossRef]

16. Opolskiene G, Sladkevicius P, Jokubkiene L, Valentin L. Threedimensional ultrasound imaging for discrimination between benign and malignant endometrium in women with postmenopausal bleeding and sonographic endometrial thickness of at least $4.5 \mathrm{~mm}$. Ultrasound Obstet Gynecol 2010;35:94-102.

17. Dueholm M, Christensen JW, Rydbjerg S, Hansen ES, Ørtoft G. Two- and three-dimensional transvaginal ultrasound with power Doppler angiography and gel infusion sonography for diagnosis of endometrial malignancy. Ultrasound Obstet Gynecol 2015;45:734-43. [CrossRef] 